\title{
Novel nonsense mutation p. Gln264Ter in the ANK1 confirms causative role for hereditary spherocytosis: a case report
}

\author{
Senmao Chai ${ }^{1}$, Rong Jiao ${ }^{2}$, Xiaodong Sun ${ }^{1}$, Pan Fu', Qiang Zhao ${ }^{1}$ and Ming Sang ${ }^{1 *}$ (D)
}

\begin{abstract}
Background: Hereditary spherocytosis (HS) is the most common haemolytic anaemia caused by congenital membrane defects of red blood cells. The name derives from the presence of spherical red blood cells in the peripheral blood. Clinical manifestations of HS are anaemia, haemolytic jaundice, and large spleen, and infection can worsen the condition, often with cholelithiasis. HS is mainly caused by abnormal functions of the products of six genes. Splenectomy is the main treatment for HS.

Case presentation: Half a day after birth, the proband exhibited HS-related symptoms, with progressive aggravation. Routine examination in the outpatient department showed an increase in white blood cells and a decrease in red blood cells. His mother had HS and a partial splenectomy. We suspected that the infant might also have HS. Genomic DNA samples were extracted from the three members of the HS trio pedigree, and genomic whole-exome sequencing (WES) was performed. The three DNA samples were amplified by polymerase chain reaction (PCR), followed by Sanger sequencing to identify mutation sites. A novel nonsense heterozygous mutation, c.790C > T (p. Gln264Ter), in the ANK1 gene, which causes premature termination of translation, was found in this Chinese family with autosomal dominant HS.
\end{abstract}

Conclusions: This de novo nonsense mutation can cause the onset of HS in early childhood, with severe symptoms. Expanding the ANK1 genotype mutation spectrum will lay a foundation for the further application of mutation screening in genetic counselling.

Keywords: Hereditary spherocytosis, Whole-exome sequencing, ANK1, Nonsense mutation

\footnotetext{
*Correspondence: smxd2000@126.com

'Hubei Institute of Parkinson's Disease at Xiangyang No.1 People's Hospital, Hubei Key Laboratory of Wudang Local Chinese Medicine Research, Hubei University of Medicine, Shiyan 442000, People's Republic of China Full list of author information is available at the end of the article
}

(c) The Author(s). 2020 Open Access This article is licensed under a Creative Commons Attribution 4.0 International License, which permits use, sharing, adaptation, distribution and reproduction in any medium or format, as long as you give appropriate credit to the original author(s) and the source, provide a link to the Creative Commons licence, and indicate if changes were made. The images or other third party material in this article are included in the article's Creative Commons licence, unless indicated otherwise in a credit line to the material. If material is not included in the article's Creative Commons licence and your intended use is not permitted by statutory regulation or exceeds the permitted use, you will need to obtain permission directly from the copyright holder. To view a copy of this licence, visit http://creativecommons.org/licenses/by/4.0/. The Creative Commons Public Domain Dedication waiver (http://creativecommons.org/publicdomain/zero/1.0/) applies to the data made available in this article, unless otherwise stated in a credit line to the data. 


\section{Background}

Hereditary spherocytosis (HS, OMIM:182900), also known as congenital hemolytic anemia, refers to one type of heterogeneous inherited anaemia characterized by the presence of spherocytes on the peripheral blood smear [1]. HS occurs all over the world, affecting approximately 1/5000 people in Europe and North America [2,3]. The main result is defects in the erythrocyte membrane skeleton, resulting in decreased surface area and spherical changes; these changes lead to loss of erythrocyte membrane elasticity and mechanical stability and difficulty in passing through the splenic capillaries, promoting erythrocyte clearance by the spleen [4]. The increase in red blood cell destruction leads to increases in bilirubin levels and causes yellowing of the skin and sclera. The spleen also grows larger due to the destruction of red blood cells and the stimulation of proliferation, followed by liver enlargement. Therefore, anaemia, jaundice, large liver and large spleen are the main symptoms of HS. Currently, the main clinical treatment is partial or total splenectomy and splenic artery embolization. Theoretically, splenic circulation is not fully developed until after birth, resulting in enhanced destruction of the globular cells surrounding the circulation and increased haemolysis. Occasional visits to the hospital cause a high rate of misdiagnosis and delayed treatment, which threatens the life of patients with HS [4]. At present, 6 abnormal genes which lead to HS have been identified. They are ANK1(ankyrin; MIM: 182900), SPTB (beta-spectrin; MIM: 616649), SPTA1(alpha-spectrin; MIM: 270970), SLC4A1(Band 3; MIM; 612,653) EPB41(protein 4.1; Phenotype MIM:611804) and EPB42(protein 4.2;MIM: 612690) [5]. There exist two genetic inheritance patterns for HS: autosomal dominant (AD) (75\%) and autosomal recessive (AR) (25\%) [2]. HS-causative mutations in SPTB and SLC4A1 show AD, whereas those in SPTA1, EPB41and $E P B 42$ show AR; alterations in ANK1 display AD or AR [2]. Although splenectomy greatly helps patients with HS, it also produces many complications. Some patients even die of infection of mesentery or portal vein occlusion after splenectomy. The most common complication is infection, especially among infants and young children.

In this study, we collected a trio pedigree of HS. The proband showed HS-related symptoms half a day after birth. Through gene sequencing, we identified a de novo nonsense variant in ANK1 in this family, promoting further application of mutation screening in genetic counselling.

\section{Case presentation}

\section{Ethical compliance and human subjects}

The study was approved by the Ethics Committee of Xiangyang No.1 People's Hospital, Hubei. Informed consent was obtained directly from the participants or parents/legal guardians of any participant under the age of 18. Blood specimens were collected from the proband and his parents. The proband was a male infant born at full term with an uneventful pregnancy. Apgar scores were $9 / 1 \mathrm{~min}$ and $10 / 5 \mathrm{~min}$. The proband showed HSrelated symptoms with gradual aggravation within half a day after birth. Routine outpatient department examination showed an increased white blood cell count and a decreased red blood cell count. The proband's mother had a history of HS and underwent partial splenectomy. We suspected the son might have HS. The probability of triggering haemolysis and entire haemolysis is increased in patients with erythrocyte brittleness (Table 1). Based on differential diagnosis, physiological jaundice, neonatal ABO haemolysis, neonatal sepsis, neonatal hepatitis syndrome and bilirubin encephalopathy were all excluded. Amoxicillin and clavulanate potassium were given for anti-infection treatment. The patient was treated with hydration, phototherapy, sodium bicarbonate, and vitamin K1 supplementation. Neonatal diseases are treated in the intensive care unit (ICU) of our hospital, with mother-infant separation.

\section{Whole-exome sequencing (WES) and sanger sequencing validation}

Genomic DNA samples from each member of the HS trio pedigree (II:1, in Fig. 1a) were extracted from whole blood and used to sequence the whole exome. The BGI Exome V4 kit (BGI) was used for in-solution enrichment of coding exons and flanking intronic sequences, as per the manufacturer's standard protocol. We used cutadapt (v1.15) to trim adaptor sequences at the tail of the sequencing reads and then aligned the sequencing reads to the human reference genome (UCSC hg19) with BWA (v0.7.15). Duplicated reads marked by Picard (v2.4.1). Qualimap [6] (v2.2.1) was used to calculate base quality metrics, the genome mapping rate, and the coverage of targeted regions. Base quality score recalibration, indel realignment and variant (SNV \& InDel) calling were performed following the best practice protocol of Genome Analysis Toolkit (GATK, v3.8). Variant filtering was performed by a finely tuned in-house script. Pass-filter variants were annotated by the Pubvar variant annotation engine (www.pubvar.com) and VEP [7]. Variant models for dominant inheritance and recessive inheritance were separately identified in the genetic analysis. Variants that met any of the following criteria were excluded from the analysis: maximum population frequency larger than 0.01 , low genotype confidence, or predicted as benign by all five algorithms, i.e., SIFT [8], PolyPhen 2 [9], MetaSVM [10], MCAP [11] and MutationTaster [12]. The pathogenic evidences of candidate disease-causing variants were scored by InterVar [13] (1.0.8) according to American College of Medical Genetics and Genomics (ACMG) guidelines [14]. Subsequently, all variants in previously reported HS genes (ANK1, SPTB, 
Table 1 Abnormal examination results on admission

\begin{tabular}{llll}
\hline Test item & Result & Cue & reference range \\
\hline Erythrocyte & $3.26 \times 10^{12} / \mathrm{L}$ & $\downarrow$ & $(5.20-6.40) \times 10^{12} / \mathrm{L}$ \\
Hemoglobin & $112.0 \mathrm{~g} / \mathrm{L}$ & $\downarrow$ & $180.0-190.0 \mathrm{~g} / \mathrm{L}$ \\
Neutrophils percentage & $79.2 \%$ & $\uparrow$ & $31.0-40.0 \%$ \\
Lymphocyte percentage & $13.6 \%$ & $\downarrow$ & $40.0-60.0 \%$ \\
Red cell distribution width & $19.0 \%$ & $\uparrow$ & $11.5-14.5 \%$ \\
Red cell distribution width standard deviation & $66.7 \mathrm{fL}$ & $\uparrow$ & $35.0-56.0 \mathrm{fL}$ \\
Began hemolysis in patients with erythrocyte brittleness & $5.40 \mathrm{~g} / \mathrm{L}$ & $\uparrow$ & $3.8-4.6 \mathrm{~g} / \mathrm{L}$ \\
Entirely hemolysis in patients with erythrocyte brittleness & $4.32 \mathrm{~g} / \mathrm{L}$ & $\uparrow$ & $2.8-3.2 \mathrm{~g} / \mathrm{L}$ \\
Total bilirubin & $194.33 \mu \mathrm{mol} / \mathrm{L}$ & $\uparrow$ & $0.0-26.0 \mu \mathrm{mol} / \mathrm{L}$ \\
Direct bilirubin & $12.47 \mu \mathrm{mol} / \mathrm{L}$ & $\uparrow$ & $0.0-8.0 \mu \mathrm{mol} / \mathrm{L}$ \\
Indirect bilirubin & $181.86 \mu \mathrm{mol} / \mathrm{L}$ & $\uparrow$ & $0.0-18 \mu \mathrm{mol} / \mathrm{L}$ \\
Y-Glutamyl transferase & $84.38 \mathrm{IU} / \mathrm{L}$ & $\uparrow$ & $10-60 \mathrm{IU} / \mathrm{L}$ \\
Alkaline phosphatase & $129.81 \mathrm{IU} / \mathrm{L}$ & $\uparrow$ & $45-125 \mathrm{IU} / \mathrm{L}$ \\
Creatine kinase & $820 \mathrm{U} / \mathrm{L}$ & $50-310 \mathrm{U} / \mathrm{L}$ \\
Creatine kinase MB isoenzyme & $44.48 \mathrm{U} / \mathrm{L}$ & $\uparrow$ & $0-24 \mathrm{U} / \mathrm{L}$ \\
Lactic dehydrogenase & $385 \mathrm{U} / \mathrm{L}$ & $120-250 \mathrm{U} / \mathrm{L}$ \\
a- Hydroxybutyrate Dehydrogenase & $281.7 \mathrm{U} / \mathrm{L}$ & $72-182 \mathrm{U} / \mathrm{L}$ \\
C- Reactive Protein & $10.75 \mathrm{mg} / \mathrm{L}$ & & $0.0-8 \mathrm{mg} / \mathrm{L}$ \\
\hline
\end{tabular}

SPTA1, SLC4A1 EPB41 and EPB42) were investigated for segregation. Finally, all variants following autosomal dominant and autosomal recessive inheritance were checked for segregation.

A novel ANK1 (NM_000037.3: c.790C > T, p. Gln264Ter) nonsense heterozygous mutation was identified in the infant and his mother. According to the evidence classification of American College of Medical Genetics and Genomics (ACMG) pathogenicity, the mutation was judged to be pathogenic (PVS1 + PM2 + PP4). Frequency data in 1000 Genomes, gnomAD exomes and East Asian gnomAD exomes were not found for this $A N K 1$ mutation site.

Primer-BLAST (https://www.ncbi.nlm.nih.gov/tools/pri mer-blast/) was used to design primers containing the mutation site: forward CAGAAATGCCCTGACAACGC and reverse ACTATTGCCTCCCACTGCAC. The annealing temperature was $60^{\circ} \mathrm{C}$, and the amplified fragment size was $395 \mathrm{bp}$. To verify the ANK1 mutation, PCR followed by Sanger sequencing were performed for the three individuals (I-1, I-2, II- 1 ) of the pedigree. Our results confirmed that a heterozygous mutation was carried by the mother (I-2) and the son (II-1, Table 2, Fig. 1b). This ANK1 nonsense heterozygous mutation (NM_000037.3: c.790C $>$ T, p. Gln264Ter) is novel and not reported or listed in the Human Gene Mutation Database (HGMD), Exome Variant Server (EVS), the 1000 Genomes databases or any other database (gnomAD, ExAC, ClinVar, dbSNP). Taken together, the causative mutation was verified by examining the trio pedigree via WES and Sanger sequencing.

\section{Discussion and conclusion}

The cytoskeleton plays a key role in maintaining the morphology and function of erythrocyte membranes. Many proteins, such as ankyrin, spectrin $\alpha$ - and $\beta$-chains, proteins 4.1 , or $4.1 \mathrm{R}$ and actin, cover the inner surface of the erythrocyte membrane to form two protein complexes, the ankyrin and protein 4.1 complex (Fig. 2). These two complexes interact to maintain the circular pie-like structure of the central fovea of red blood cells. The former complex consists of Band 3 tetramers, Rh, RhAG, CD47, Glycophorin A and Protein 4.2; the latter consists of Band 3 dimers binding Adducins $\alpha$ and $\beta$, Glycophorin $C$, GLUT1 and Stomatin $[15,16]$. ANK1, the most common of the 6 pathogenic genes of HS, is an important member of the anchoring protein family, which is mainly expressed in the erythrocyte membrane. Anchoring proteins are connected to complete membrane proteins, further connected to the spectrin and cytoskeleton. They play an important role in cell movement, activation and proliferation and participate in maintaining the specific morphology of cell membranes. Mutation of the ANK1 gene can cause red blood cells to change from a double concave disc shape to a spherical shape and reduce its deforming capacity. In capillary networks such as the liver and spleen, rupture and haemolysis may occur due to mechanical damage [17]. There are more than 60 known mutations in the human HS-related ANK1 gene, among which the most common include missense mutations, nonsense mutations, frame shifts and splice site mutations. The clinical severity of HS 


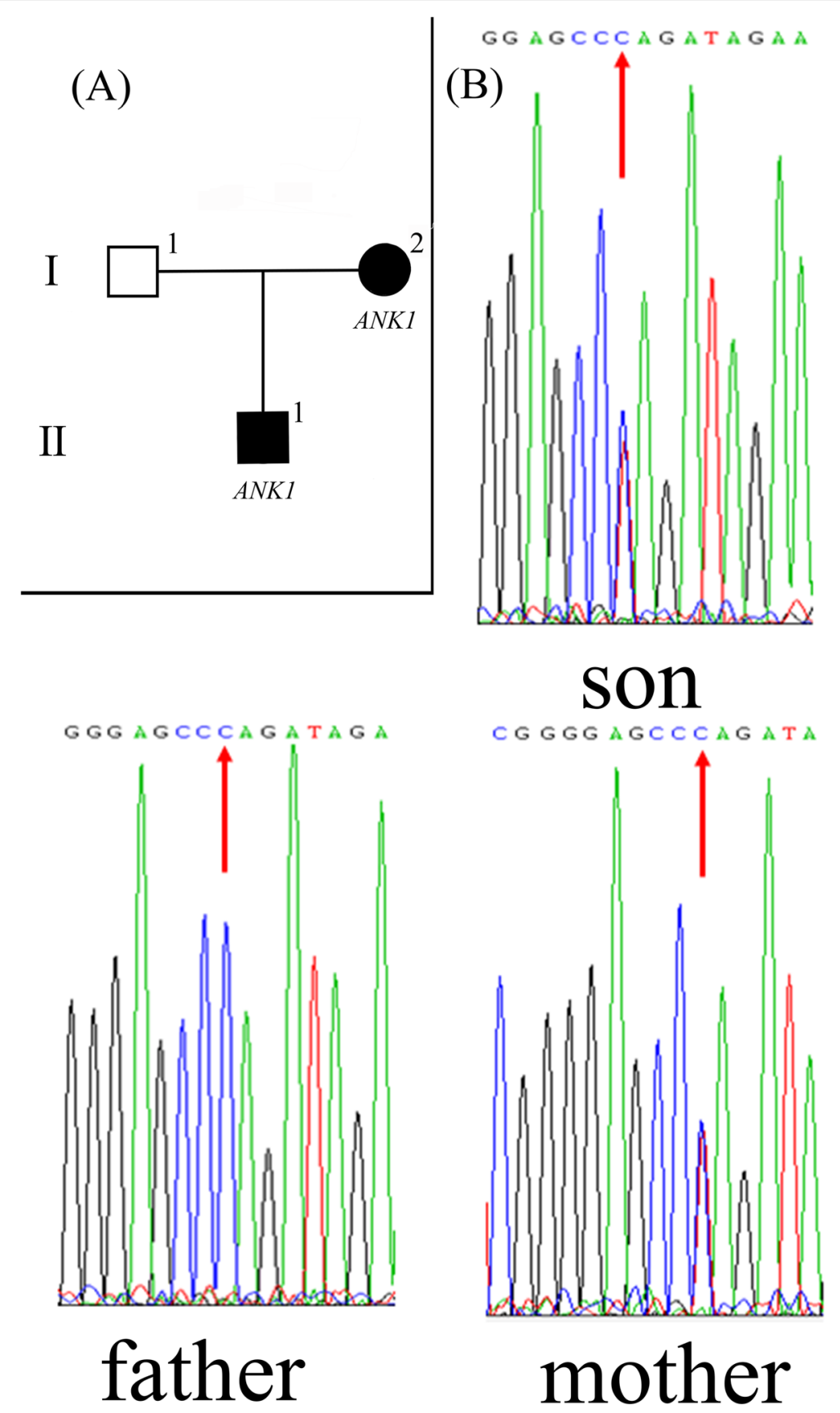

Fig. 1 The novel pathogenic variant in the ANK1 gene. a The family tree. $\mathbf{b}$ The hemizygous variant confirmed by Sanger sequencing. The heterozygous mutations has been carried by Mother and son. The red arrow indicates the mutation site

Table 2 ANK1 mutation of the family members

\begin{tabular}{llllll}
\hline Family members & Exon & Reference sequence & Type & cDNA & Protein \\
\hline Father & 8 & NM_000037.3 & Wild & c.790C & p. Gln264 \\
Mather & 8 & NM_000037.3 & Mutant & c.790C $>$ T & p. Gln264Ter \\
propositus & 8 & NM_000037.3 & Mutant & c.790C > T & p. Gln264Ter \\
\hline
\end{tabular}




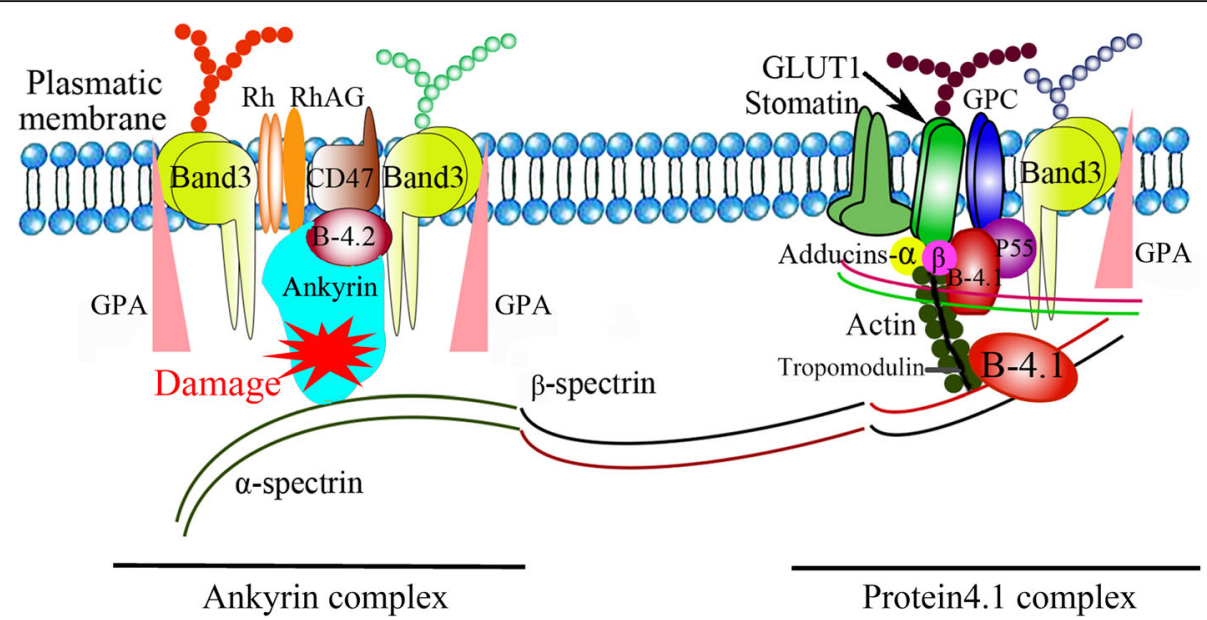

Fig. 2 A simplified cross section diagram of erythrocyte membrane. Phospholipid bilayer is the basic scaffold of red blood cell membrane. Protein Ankyrin is marked in red to indicate damage due to gene mutation. At present, it has been found that 6 proteins Ankyrin; Spectrin alpha-chain; Spectrin beta-chain; Band 3; Protein 4.1 and Protein 4.2 paly a pathogenic role in Hereditary spherocytosis. The relative positions of proteins in various complexes are mostly unknown. The shapes of the major proteins are fictitious. GPA: glycophorin A; Rh: Rhesus polypeptide; B-4.1: protein band 4.1; B-4.2: protein band 4.2; GPC: glycophorin C; RhAG: Rh-associated glycoprotein

depends on the degree of membrane loss, and most ANK1 mutations show AD inheritance [17, 18]. Ankyrin is the main component of erythrocyte membranes and composed of three structural domains: a 24 homologous repeat $\mathrm{N}$ terminal membrane-binding domain (MBD) involved in the binding of Band 3 protein; a Spectrin-binding central domain (SBD); and a least conserved carboxy-terminal regulatory domain subject to variation [19].

This clinical report shows that the proband and his mother carry a de novo nonsense heterozygous mutation in the ANK1 (NM_000037.3: c.790C > T, p. Gln264Ter) gene. In contrast to most affected newborns, this proband presented severe anaemia in a very early life and remained transfusion dependent. Half a day after birth, his skin appeared yellow dye, and suffered from progressive aggravation, his white blood cell counts and c-reactive protein both were increased significantly. Our findings show the de novo nonsense mutation can cause the onset of the disease in early childhood, even with more severer symptoms. Expanding the genotypic mutation spectrum of $A N K 1$, can work for further application of mutation screening in the genetic counseling.

\section{Abbreviations}

HS: Hereditary spherocytosis; AD: Autosomal dominant; AR: Autosomal recessive; ANK1: Ankyrin 1; SPTB: Beta-spectrin; SPTA1: Alpha-spectrin; SLC4A1: Band 3; EPB41: Protein 4.1; EPB42: Protein 4.2; WES: Whole exome sequencing; ACMG: American College of Medical Genetics and Genomics

\section{Acknowledgements}

We thank the patient's family for participating in this study, Kd of Reproductive Medicine Center, Renmin Hospital, Hubei University of Medicine. We thank all the staff of Hubei Institute of Parkinson's Disease at Xiangyang No.1 People's Hospital. They were very helpful.

\section{Authors' contributions}

MS, Sm C and RJ Conceived and designed the experiments. Sm C and, Xd S Performed the sample recruitment and Whole exome and sanger sequencing. QZ and PF Conducted the data analysis. MS Provided the reagents/ materials/ analysis tools to carry out the experiments. Sm C Wrote the paper. All authors read and approved the final manuscript.

\section{Funding}

This study was funded by the Natural Science Foundation of Hubei Province. Award Number: 2019AHB068, 2017CFB674,2018CFB701. Open Project of Hubei Key Laboratory of Wudang Local Chinese Medicine Research. Award Number: WDCM2018009. The Innovative Team Project from the Institute of Medicine and Nursing at Hubei University of Medicine. Award Number: 2017YHKT02. The recipient is Ming Sang for All of the funding.MS is also the corresponding author

The funding bodies played no role in the design of the study and collection, analysis, and interpretation of data and in writing the manuscript.

\section{Availability of data and materials}

All relevant data are within the paper, including raw data from WES, Further information is available from the authors on request. All raw data related to our study has been submitted on NCBI Sequence Read Archive (SRA) (SRA RunSelector: https://www.ncbi.nlm.nih.gov/sra/PRJNA671560). Accession number is PRJNA671560. Reference datasets were listed in the "Wholeexome sequencing (WES) and Sanger sequencing validation" section, such as the human reference genome (UCSC hg19) and transcription number (NM_000037.3).

\section{Ethics approval and consent to participate}

The Ethical approval for the study was taken from Ethics Committee of Xiangyang No.1 People's Hospital. Written informed consents were obtained from the parents or guardians of the propositus and 74 healthy control cases.

\section{Consent for publication}

Written informed consent for publication of individual/genetic data was obtained from the participants or parents/legal guardians of any participant under the age of 18.

\section{Competing interests}

The authors declare no competing interest. 


\section{Author details}

'Hubei Institute of Parkinson's Disease at Xiangyang No.1 People's Hospital, Hubei Key Laboratory of Wudang Local Chinese Medicine Research, Hubei University of Medicine, Shiyan 442000, People's Republic of China.

${ }^{2}$ Department of New pediatric, Xiangyang No.1 People's Hospital, Hubei University of Medicine, Xiangyang, Hubei, China.

Received: 4 July 2020 Accepted: 29 October 2020

\section{Published online: 13 November 2020}

\section{References}

1. Eber S, Lux SE. Hereditary spherocytosis--defects in proteins that connect the membrane skeleton to the lipid bilayer. Semin Hematol. 2004;41(2):118-41.

2. Perrotta S, Gallagher PG, Mohandas N. Hereditary spherocytosis. Lancet. 2008:372(9647):1411-26.

3. Wang C, Cui Y, Li Y, Liu X, Han J. A systematic review of hereditary spherocytosis reported in Chinese biomedical journals from 1978 to 2013 and estimation of the prevalence of the disease using a disease model. Intractable Rare Dis Res. 2015;4(2):76-81.

4. Manciu S, Matei E, Trandafir B. Hereditary Spherocytosis - Diagnosis, Surgical Treatment and Outcomes. A Literature Review. Chirurgia (Bucur). 2017; 112(2):110-6.

5. Delaunay J. Molecular basis of red cell membrane disorders. Acta Haematol. 2002;108(4):210-8.

6. Garcia-Alcalde F, Okonechnikov K, Carbonell J, Cruz LM, Gotz S, Tarazona S, Dopazo J, Meyer TF, Conesa A. Qualimap: evaluating next-generation sequencing alignment data. Bioinformatics. 2012;28(20):2678-9.

7. McLaren W, Gil L, Hunt SE, Riat HS, Ritchie GR, Thormann A, Flicek P, Cunningham F. The Ensembl variant effect predictor. Genome Biol. 2016; 17(1):122.

8. Kumar P, Henikoff S, Ng PC. Predicting the effects of coding nonsynonymous variants on protein function using the SIFT algorithm. Nat Protoc. 2009:4(7):1073-81.

9. Adzhubei IA, Schmidt S, Peshkin L, Ramensky VE, Gerasimova A, Bork P, Kondrashov AS, Sunyaev SR. A method and server for predicting damaging missense mutations. Nat Methods. 2010;7(4):248-9.

10. Kim S, Jhong JH, Lee J, Koo JY. Meta-analytic support vector machine for integrating multiple omics data. BioData Mining. 2017;10:2.

11. Jagadeesh KA, Wenger AM, Berger MJ, Guturu H, Stenson PD, Cooper DN, Bernstein JA, Bejerano G. M-CAP eliminates a majority of variants of uncertain significance in clinical exomes at high sensitivity. Nat Genet. 2016; 48(12):1581-6.

12. Schwarz JM, Rodelsperger C, Schuelke M, Seelow D. MutationTaster evaluates disease-causing potential of sequence alterations. Nat Methods. 2010;7(8):575-6.

13. Li Q, Wang K. InterVar: clinical interpretation of genetic variants by the 2015 ACMG-AMP guidelines. Am J Hum Genet. 2017;100(2):267-80.

14. Richards S, Aziz N, Bale S, Bick D, Das S, Gastier-Foster J, Grody WW, Hegde $M$, Lyon E, Spector E, et al. Standards and guidelines for the interpretation of sequence variants: a joint consensus recommendation of the American College of Medical Genetics and Genomics and the Association for Molecular Pathology. Genet Med. 2015;17(5):405-24.

15. Bruce L, Beckmann R, Ribeiro ML, Peters LL, Chasis JA, Delaunay J, Mohandas N, Anstee DJ, Tanner MJ. A band 3-based macrocomplex of integral and peripheral proteins in the RBC membrane. Blood. 2003;101(10):4180-8.

16. Mouro-Chanteloup I, Delaunay J, Gane P, Nicolas V, Johansen M, Brown EJ, Peters LL, Van Kim CL, Cartron JP, Colin Y. Evidence that the red cell skeleton protein 4.2 interacts with the Rh membrane complex member CD47. Blood. 2003;101(1):338-44.

17. He BJ, Liao L, Deng ZF, Tao YF, Xu YC, Lin FQ. Molecular genetic mechanisms of hereditary spherocytosis: current perspectives. Acta Haematol. 2018;139(1):60-6.

18. Park J, Jeong DC, Yoo J, Jang W, Chae H, Kim J, Kwon A, Choi H, Lee JW, Chung NG, et al. Mutational characteristics of ANK1 and SPTB genes in hereditary spherocytosis. Clin Genet. 2016;90(1):69-78.

19. Andolfo I, Russo R, Gambale A, lolascon A. New insights on hereditary erythrocyte membrane defects. Haematologica. 2016;101(11):1284-94.

\section{Publisher's Note}

Springer Nature remains neutral with regard to jurisdictional claims in published maps and institutional affiliations.

Ready to submit your research? Choose BMC and benefit from:

- fast, convenient online submission

- thorough peer review by experienced researchers in your field

- rapid publication on acceptance

- support for research data, including large and complex data types

- gold Open Access which fosters wider collaboration and increased citations

- maximum visibility for your research: over $100 \mathrm{M}$ website views per year

At BMC, research is always in progress.

Learn more biomedcentral.com/submissions 